\title{
A genome-wide screen for modifiers of transgene variegation identifies genes with critical roles in development
} Alyson Ashe*, Daniel K Morgan ${ }^{*+}$, Nadia C Whitelaw ${ }^{*+}$, Timothy J Bruxner*, Nicola K Vickaryous*, Liza L Cox ${ }^{\ddagger}$, Natalie C Butterfield§, Carol Wicking§, Marnie E Blewitt and Emma Whitelaw*

\begin{abstract}
Addresses: *Epigenetics Laboratory, Queensland Institute of Medical Research, 300 Herston Road, Herston, Queensland 4006, Australia. ${ }^{+}$School of Medicine, University of Queensland, Brisbane, 4001, Australia. ${ }^{*}$ Division of Craniofacial Medicine, Department of Pediatrics, University of Washington, Seattle 98195, WA, USA. §Institute for Molecular Bioscience, The University of Queensland, St Lucia, Queensland 4072, Australia. `Walter and Eliza Hall Institute, Melbourne, Victoria 3050, Australia. ${ }^{¥}$ Iron Metabolism Laboratory, Queensland Institute of Medical Research, 300 Herston Road, Herston, Queensland 4006, Australia.
\end{abstract}

Correspondence: Emma Whitelaw. Email: emma.whitelaw@qimr.edu.au

Published: 19 December 2008

Genome Biology 2008, 9:RI82 (doi:10.1 186/gb-2008-9-12-r182)

The electronic version of this article is the complete one and can be found online at http://genomebiology.com/2008/9/I2/R I82
Received: 21 June 2008

Revised: 22 October 2008

Accepted: 19 December 2008

(c) 2008 Ashe et al.; licensee BioMed Central Ltd.

This is an open access article distributed under the terms of the Creative Commons Attribution License (http://creativecommons.org/licenses/by/2.0), which permits unrestricted use, distribution, and reproduction in any medium, provided the original work is properly cited.

\begin{abstract}
Background: Some years ago we established an N-ethyl-N-nitrosourea screen for modifiers of transgene variegation in the mouse and a preliminary description of the first six mutant lines, named MommeDI-D6, has been published. We have reported the underlying genes in three cases: MommeDI is a mutation in SMC hinge domain containing I (SmchdI), a novel modifier of epigenetic gene silencing; MommeD2 is a mutation in DNA methyltransferase I (Dnmtl); and MommeD4 is a mutation in Smarca 5 (Snf2h), a known chromatin remodeler. The identification of Dnmtl and Smarca5 attest to the effectiveness of the screen design.

Results: We have now extended the screen and have identified four new modifiers, MommeD7D 10. Here we show that all ten MommeDs link to unique sites in the genome, that homozygosity for the mutations is associated with severe developmental abnormalities and that heterozygosity results in phenotypic abnormalities and reduced reproductive fitness in some cases. In addition, we have now identified the underlying genes for MommeD5 and MommeDI0. MommeD5 is a mutation in $\mathrm{Hdacl}$, which encodes histone deacetylase I, and MommeDIO is a mutation in Baz Ib (also known as Williams syndrome transcription factor), which encodes a transcription factor containing a PHDtype zinc finger and a bromodomain. We show that reduction in the level of Bazlb in the mouse results in craniofacial features reminiscent of Williams syndrome.

Conclusions: These results demonstrate the importance of dosage-dependent epigenetic reprogramming in the development of the embryo and the power of the screen to provide mouse models to study this process.
\end{abstract}




\section{Background}

Random mutagenesis screens for modifiers of position effect variegation were initiated in Drosophila in the 1960 s $[1,2]$. The screens used a fly strain, called $w^{v}$, that displays variegated expression of the white $(w)$ locus resulting in red and white patches in the eye. Variegation refers to the 'salt and pepper' expression of some genes due to the stochastic establishment of an epigenetic state at their promoters. The best characterized example of variegation in mammals is the coat color of mice carrying the $A^{v y}$ allele [3,4]. In this case there is a correlation between DNA methylation at the promoter and silencing of the gene [5,6]. Alleles of this type provide us with an opportunity to study epigenetic gene silencing at a molecular level in a whole organism.

The extent of the variegation at the $w^{v}$ locus, that is, the ratio of red to white patches in the eye, was known to be sensitive to strain background, suggesting the existence of genetic modifiers. Offspring of mutagenized flies were screened for changes in the degree of variegation. These screens have been continued to saturation and the results suggest that there are between 100 and 150 such genes [7,8]. Approximately onethird of these have been identified and, as expected, most turn out to play critical roles in epigenetic gene silencing [1,9]. These include genes encoding proteins involved in heterochromatin formation, for example, HP1 and histone methyltransferases [8].

Recently, we established a similar screen in the mouse using a transgenic line that expresses green fluorescent protein (GFP) in a variegated manner in erythrocytes [10]. We anticipated that the screen would produce mutant lines that would help clarify the role of epigenetic gene silencing in mammals. Offspring of N-ethyl-N-nitrosourea (ENU) treated males were screened for changes in the percentage of erythrocytes expressing GFP (measured by flow cytometry). In those individuals in which the percentage of expressing cells was higher or lower than the wild-type mean by more than two standard deviations, heritability was tested. A preliminary description of the first six heritable mutations, which we refer to as Modifiers of murine metastable epialleles or Mommes, following the screening of $608 \mathrm{G1}$ offspring, has been published [10]. We reported that all six were dosage-effect genes and five of the six were homozygous lethal, with loss of homozygotes apparent at weaning, but no knowledge of when death occurred. At the time of publication in 2005, none of the underlying genes had been identified. Since then we have identified the underlying mutation in three cases, MommeD1, MommeD2 and MommeD4. MommeD1 is a mutation in SMC hinge domain containing 1 (SmcHD1), encoding a previously uncharacterized protein containing a domain normally found in SMC proteins and we have gone on to show that this protein has a critical role in X inactivation [11]. MommeD2 is a mutation in DNA methyltransferase 1, Dnmt1, encoding a DNA methyltransferase, and MommeD4 is a mutation in Smarca5, encoding Snf2h, a chromatin-remodeling enzyme
[12]. The finding of Dnmt1 and Smarca5, both known to be involved in epigenetic reprogramming, validates the screen. Here we report an update of the screen, adding four new MommeDs, identifying the underlying point mutation in two more cases, and further characterizing the phenotypes associated with hetero- and homozygosity.

\section{Results and discussion Integration site of the GFP transgene}

We have previously reported that the GFP transgene used in this screen has integrated as an approximately 11 copy array on chromosome 1 [10]. We were keen to further characterize the integration site. PCR using primers specific to the 3 ' end of the transgene construct in combination with degenerate random tagging primers (genome walking) revealed that the transgene had integrated into chromosome 1 at 47,747,486 bp (UCSC web browser, July 2007 assembly). This site of integration is neither centromeric nor telomeric, and so presumably the silencing is related to the multicopy nature of the transgene array $[13,14]$. The integration site does not disrupt any annotated genes, and is approximately $1 \mathrm{Mb}$ from the closest annotated transcript.

\section{The identification of MommeD7-DI0}

We have now screened an additional $400 \mathrm{G} 1$ offspring and recovered four more Mommes, MommeD7-D1O (Figure 1, Table 1). The fluorescence activated cell sorting (FACS)-based screening is carried out on a drop of blood taken at weaning, using a gate set to exclude $99.9 \%$ of autofluorescing cells. Under these conditions, wild-type mice homozygous for the transgene express GFP in $55 \%$ of erythrocytes. MommeD 7 is a suppressor of variegation, that is, the percentage of cells expressing the transgene was significantly higher than it was in wild-type individuals (Table 1). MommeD8, $D 9$ and $D 1 O$ are enhancers of variegation, that is, the percentages of expressing cells were significantly lower than they were in wild-type individuals (Table 1). The mean fluorescence level in expressing cells also changed. We have reported previously that as the percentage of expressing cells increases, the mean fluorescence of the expressing cells increases [10]. We presume that since the mice are homozygous for the GFP transgene, this is mainly due to an increase in the proportion of expressing cells with two active GFP alleles. However, in the case of MommeD7 the level was more than double that seen in the wild-type littermates. We hypothesized that this was likely to be the consequence of an increase in the percentage of reticulocytes in the peripheral blood of this mutant, as mature red cells, with no ability to produce new proteins, would have, on average, less GFP than reticulocytes (see below). In the cases of MommeD8, D9 and D1o the mean fluorescence levels were slightly lower than that seen in the wildtype littermates, consistent with a presumed reduction in the proportion of cells with two active GFP alleles. 

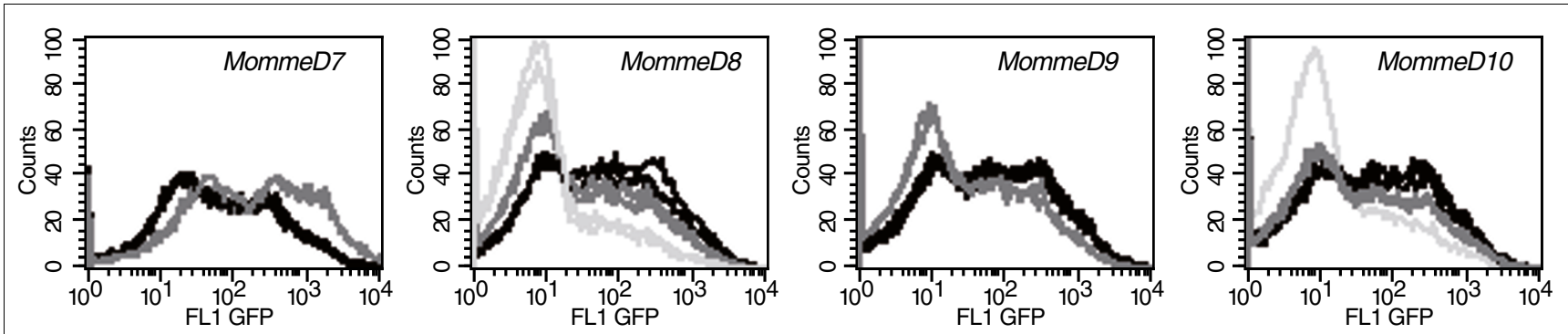

\section{Figure I}

GFP expression profiles in MommeD7-D I0. Erythrocytes from 3-week-old mice were analyzed by flow cytometry. In each case, the expression profiles from one litter of a heterozygous intercross are displayed. The phenotypically wild-type mice are shown in black, heterozygotes in dark grey, and homozygotes (MommeD8 and DIO only) in light grey. The $\mathrm{x}$-axis represents the erythrocyte fluorescence on a logarithmic scale, and the $y$-axis is the number of cells detected at each fluorescence level. For quantitative and statistical significance, see Table I.

For each MommeD, the heritability of the mutation has been tested and confirmed over at least 5 generations by using at least 30 litters. During the breeding of each mutant line, the expression profiles remained constant, consistent with the idea that we were following a single mutation in each case. The frequency with which we found these mutations, 1 in 100 G1 offspring, was similar to our previous results [10].

\section{Homozygous lethality}

Following heterozygous intercrosses, the proportion of expression types observed in the offspring at weaning was consistent with a semidominant homozygous lethal mutation in the cases of MommeD7 and MommeD9, since only two GFP expression profiles were observed (Figure 1, Tables 1 and 2) and there was a significant litter size reduction in both cases (Figure 2). In the cases of MommeD8 and MommeD1O, three expression profiles were observed, suggesting viability of some homozygotes (Figure 1, Tables 1 and 2) and in the case of MommeD1o this was later confirmed by genotyping for the point mutation. In both cases, fewer individuals with the severe phenotypes were observed than predicted by Mendelian inheritance, suggesting reduced viability of the homozygotes (Table 2). This conclusion is supported by significant litter size reductions in both cases (Figure 2). There is also a suggestion of some heterozygous death in the case of MommeD8 and, to a lesser extent, MommeD1o but this is not statistically significant.

\section{Homozygous lethality occurs at different stages of development}

Litter size reductions following heterozygous intercrosses have already been reported for MommeD1-D6 at weaning [10], but the timing of the losses has only been reported for MommeD1, D2 and D4. MommeD1 is homozygous lethal in females only, with death occurring around mid-gestation $[10,11]$. MommeD2 and MommeD4 are homozygous lethal at 8.5 days post-coitus (dpc) and $17.5 \mathrm{dpc}$, respectively [10,12]. Here we describe the timing of the losses for MommeD3, $D_{5}$, D6, D7, D8, D9 and D1o.

Following intercrosses between $\mathrm{MommeD}^{-/+}$(genotypes determined by GFP fluorescence and progeny testing), dissections at $14.5 \mathrm{dpc}$ suggested that death of homozygotes had already occurred (Table 3). This was confirmed following a FVB/C $57 \mathrm{~F}_{1}$ MommeD $3^{-/+}$intercross, where embryos could be genotyped using microsatellite markers across the linked interval (Table 3). These data suggest $\mathrm{MommeD}_{3} \mathrm{~T}^{-/}$mice were dying at or before $14.5 \mathrm{dpc}$. Similar results were obtained for

Table I

Quantitative analysis of GFP expression following heterozygous intercrosses

\begin{tabular}{|c|c|c|c|c|c|c|}
\hline \multirow[b]{2}{*}{ Mutant } & \multicolumn{3}{|c|}{ Percentage expressing cells } & \multicolumn{3}{|c|}{ Mean fluorescence } \\
\hline & WT (n) & Heterozygote (n) & Homozygote (n) & WT (n) & Heterozygote (n) & Homozygote $(n)$ \\
\hline MommeD7 & $55 \pm 7(7)$ & $80 \pm 3^{*}(13)$ & NA & $286 \pm 43(7)$ & $700 \pm 52 *(13)$ & NA \\
\hline MommeD8 & $54 \pm 4(40)$ & $4 I \pm 4 *(55)$ & $15 \pm 4 *(15)$ & $287 \pm 25(40)$ & $244 \pm 28^{*}(55)$ & $161 \pm 27^{*}(15)$ \\
\hline MommeD9 & $59 \pm 4(8)$ & $39 \pm 6 *(13)$ & NA & $326 \pm 37(8)$ & $228 \pm 27^{*}(13)$ & NA \\
\hline MommeDIO & $55 \pm 4(46)$ & $44 \pm 3^{*}(87)$ & $22 \pm 2 *(6)$ & $295 \pm 33(46)$ & $269 \pm 26 *(87)$ & $243 \pm 19 *(6)$ \\
\hline
\end{tabular}

The percentage of expressing cells was determined by using a GFP+ gate, which was set to exclude $99.9 \%$ of wild-type (WT) cells. Data were collected from at least four litters in each case. The data for the MommeD 9 colony were collected using a different laser. Each mutant line has a significantly different expression profile to that of wild-type littermates, reproducible over many generations. $*_{p} \leq 0.000 \mathrm{I}$. 


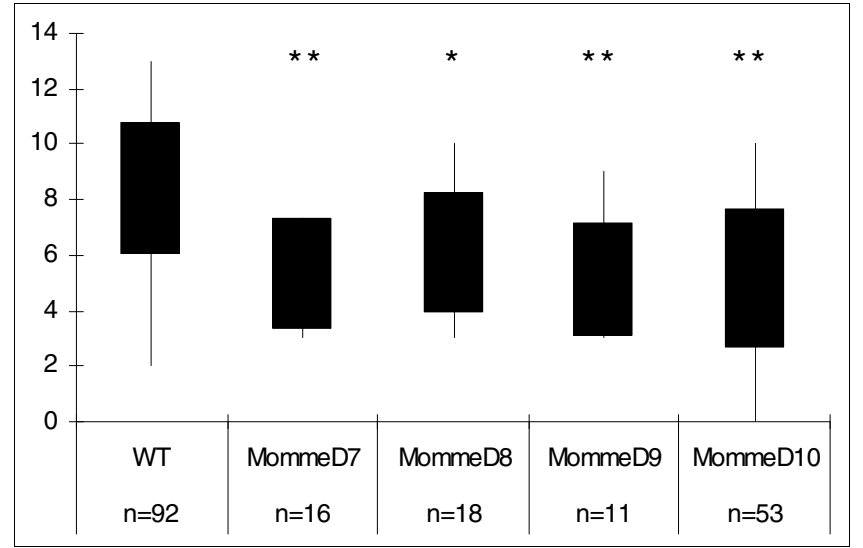

Figure 2

Litter size at weaning following heterozygous intercrosses. Litter size at weaning in all of MommeD7-DIO is significantly lower than that found in a wild-type (WT) cross. $n$ represents the number of litters. ${ }^{*} p<$ $0.05 ; * * p<0.001$

MommeD5 at 14.5 dpc (Table 3). Once the MommeD5 point mutation had been found (see below), these crosses were repeated and dissections were performed at 10.5 dpc. Again, a significantly higher than expected proportion of developmentally delayed embryos were detected (Table 3). These embryos were genotyped and found to be MommeD5-/- in all cases, indicating developmental arrest at around 8-9 dpc.
Results obtained for MommeD6 (genotypes determined by GFP fluorescence and progeny testing) were similar (Table 3), suggesting MommeD6-/- embryos arrest around 8-9 dpc.

Following MommeD $7^{/+}$intercrosses (genotypes determined by GFP fluorescence and progeny testing), a small but significant increase in abnormal embryos was detected at $14.5 \mathrm{dpc}$ (Table 3). This increase is not enough to account for all expected MommeD $7^{/-}$mice. At $17.5 \mathrm{dpc}$, approximately onequarter of the embryos were pale (Table 3, Figure 3a), suggesting a red cell defect in the homozygotes. Homozygous MommeD7 mutants were never seen at weaning (Table 2), and preliminary observations suggest that they die in the first few days after birth. Further analysis of adult heterozygous individuals revealed severe splenomegaly (Figure $3 \mathrm{~b}$ ) and a marked increase in reticulocytes in peripheral blood (Figure 3c).

We hypothesized that this increase in reticulocytes was responsible for the larger than expected increase in the average fluorescence level of the GFP transgene in expressing cells observed in this line (Table 1). We performed FACS analysis on whole blood after staining for reticulocytes with propidium iodide. As expected, MommeD $\gamma^{1+}$ mice had a threefold increase in the percentage of reticulocytes compared to MommeD $7^{+/+}$mice (Figure $3 \mathrm{~d}$ ), and the percentage of GFP fluorescence in both MommeD $7^{-/+}$and $M o m m e D 7^{+/+}$was higher in reticulocytes than mature red cells (Figure $3 \mathrm{e}$ ).

Table 2

\begin{tabular}{|c|c|c|c|}
\hline & Observed & Expected if semidominant, homozygous viable & Expected if semidominant, homozygous lethal \\
\hline \multicolumn{4}{|l|}{ MommeD7 } \\
\hline Heterozygote* & 65 & & 60 \\
\hline Wild type & 25 & & 30 \\
\hline \multicolumn{4}{|l|}{ MommeD8 } \\
\hline Homozygote* & 14 & 27.5 & \\
\hline Heterozygote* & 56 & 55 & \\
\hline Wild type & 40 & 27.5 & \\
\hline \multicolumn{4}{|l|}{ MommeD9 } \\
\hline Heterozygote* & 31 & & 37.3 \\
\hline Wild type & 25 & & 18.7 \\
\hline \multicolumn{4}{|l|}{ MommeDIO } \\
\hline Homozygote* $\dagger$ & 14 & 77.25 & \\
\hline Heterozygote* $\dagger$ & 187 & 154.5 & \\
\hline Wild type & 108 & 77.25 & \\
\hline
\end{tabular}


Table 3

\begin{tabular}{|c|c|c|c|c|c|c|}
\hline \multirow[b]{2}{*}{ Name } & \multicolumn{2}{|r|}{$10.5 \mathrm{dpc}$} & \multicolumn{2}{|r|}{$14.5 \mathrm{dpc}$} & \multicolumn{2}{|c|}{$17.5 / 18.5 \mathrm{dpc}$} \\
\hline & Normal & Abnormal or resorbed & Normal & Abnormal or resorbed & Normal & Abnormal or resorbed \\
\hline MommeD3 & & & $48(60 \%)$ & $32(40 \%)^{*}$ & & \\
\hline MommeD3 $(F I)$ & & & $80(74 \%)$ & $28(26 \%)+$ & & \\
\hline MommeD5 & $35(74 \%)$ & $12(26 \%)^{*} \ddagger$ & $42(72 \%)$ & $16(28 \%)^{*}$ & & \\
\hline MommeD6 & $38(63 \%)$ & $22(37 \%)^{*}$ & $25(62.5 \%)$ & $15(37.5 \%)^{*}$ & & \\
\hline MommeD7 & & & $44(85 \%)$ & $8(15 \%)^{\S}$ & $63(74 \%)$ & $22(26 \%)$ \\
\hline MommeD8 & & & 101 (94\%) & $7(6 \%)$ & & \\
\hline MommeD9 & $16(64 \%)$ & $9(36 \%)^{*}$ & $19(59 \%)$ & $13(4 \mid \%)^{*}$ & & \\
\hline MommeDIO & & & $54(93 \%)$ & $4(7 \%)$ & $86(88 \%)^{\top}$ & $12(12 \%)^{¥}$ \\
\hline Control cross & 34 (94\%) & $2(6 \%)$ & 266 (93\%) & $19(7 \%)$ & & \\
\hline
\end{tabular}

tThe abnormal embryos from which DNA could be extracted (17) were genotyped across the linked interval and all shown to be MommeD3\%/. ‡These abnormal (very small) embryos were genotyped and shown to be MommeD5-1- TThese normal embryos were genotyped and found to have

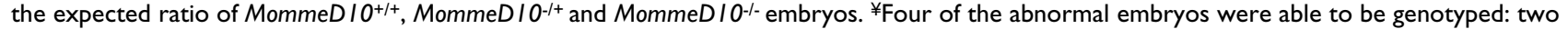
were MommeDI $0^{-/+}$and two were MommeD I $0^{-1-}$. $*_{p}<0.000 \mathrm{I} ; \S p<0.05$.

Although this is only significant for $M o m m e D 7^{-1+}(p<0.005)$, the trend is there for MommeD $7^{+/+}$mice $(p=0.07)$. This is consistent with the idea that a change in the erythroid cell populations contributes to the dramatic increase in the average fluorescence level of the GFP transgene in MommeD $7^{-{ }^{+}}$ mice.

Some MommeD8-/- mice (classified by their GFP expression profile and progeny testing) were viable at weaning but they were rare (Figure 1, Table 2). Following MommeD8-/+ intercrosses, dissections at $14.5 \mathrm{dpc}$ showed no increase in the number of abnormal or resorbed embryos (Table 3). Litter size at birth was not significantly different from that seen in wild-type litters (data not shown), suggesting that the death of most MommeD8-/- individuals occurred after birth and before weaning. The only obvious phenotypic abnormality seen in MommeD8-/- mice that survived to weaning was reduced size. MommeD8 homozygotes were significantly smaller (6.6o g \pm 0.25 standard error of the mean (SEM)) than their wild-type $(8.54 \mathrm{~g} \pm 0.33 \mathrm{SEM}, p<0.001)$ and heterozygous (8.65 $\mathrm{g} \pm 0.29 \mathrm{SEM}, p<0.0001)$ littermates.

Dissections following MommeD9-/+ (determined by GFP fluorescence and progeny testing) intercrosses revealed a pattern similar to that seen for MommeD5 and MommeD6, suggesting MommeD9-/- embryos arrest before 9.5 dpc (Table 3 ). In the case of MommeD1O the data suggest that death of homozygotes occurred after birth (Table 3), and preliminary data collected at $\mathrm{P} 7$ indicated death in the first week of life (data not shown). Some $\mathrm{MommeD}^{-/} \mathrm{O}^{-}$- individuals survived to weaning but they were extremely rare. This was confirmed by genotyping once the point mutation was identified.
So in all ten MommeDs produced so far, homozygosity for the mutation is associated with embryonic or perinatal lethality (Tables 3 and 4).

\section{Abnormal phenotypes associated with heterozygosity for MommeD7-DI 0}

Extensive phenotyping of the heterozygous MommeD mutant lines has not been carried out. However, in some cases heterozygous effects were obvious, for example, the haematopoietic defect in MommeD $7^{-/+}$mice described above. We have also noticed some litter size reduction during the breeding of these strains. The data for the breeding of MommeD7, D8, D9 and $D_{1 O}$ are shown in Figure 4. Following crosses between heterozygous males and wild-type females in the FVB strain, we found significant litter size reductions in the cases of MommeD9 and MommeD1o, but not in the cases of MommeD7 and MommeD8. A breakdown of the offspring by sex and genotype revealed that for MommeDy and MommeD1o, the litter size reduction was associated with a deviation from Mendelian patterns of inheritance $(p<0.05$ in both cases) and a reduction in the number of mutants (Figure 4). These two cases of transmission ratio distortion have not been investigated further but they do suggest that heterozygosity for the MommeD mutations is associated with some level of disadvantage. There also appears to be a skewed sex ratio in the wild-type offspring of MommeD 9 sires, suggesting the phenotype of the father can affect his wild-type offspring. While we have not characterized this in any more detail, the idea of a paternal effect is not new. We have previously published examples of paternal effects resulting from haploinsufficiency of modifiers of epigenetic gene silencing in the mouse [12]. 


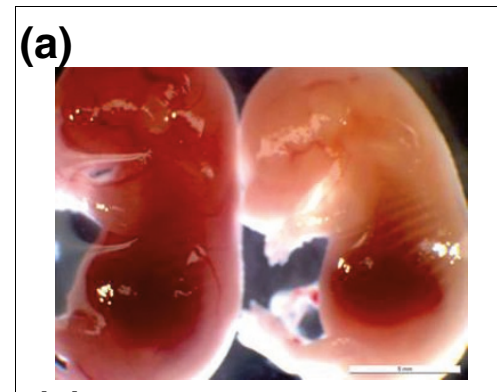

(c)
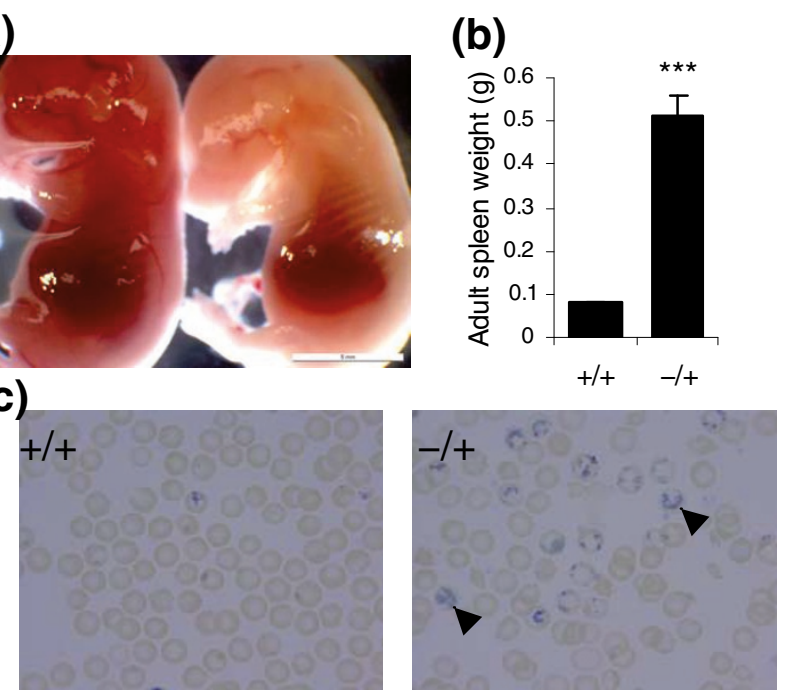

(d)
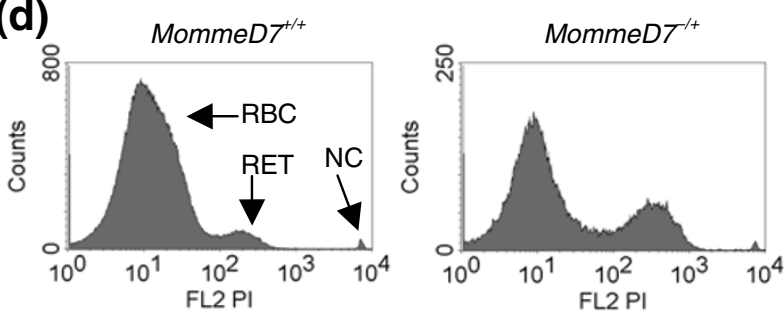

(e)

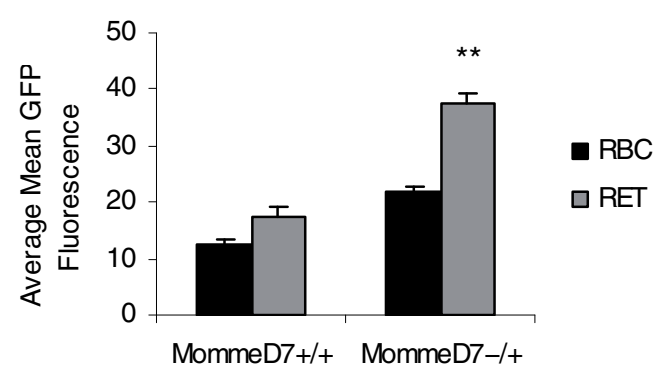

Figure 3

Hematopoietic abnormalities in MommeD7 mice. (a) Examples of phenotypically normal and abnormal (pale) embryos at $17.5 \mathrm{dpc}$. Abnormal embryos are assumed to be homozygous for MommeD7. Scale bar $=5$ $\mathrm{mm}$. (b) Spleen weights from MommeD7 $7^{+/+}$and MommeD7 $7^{-/+}$adult mice. Error bars represent SEM. *** $p<0.0001$. (c) Blood smears from MommeD $^{+/+}$(left) and MommeD7-/+ (right) mice stained for reticulocytes (shown with arrowheads). (d) Representative histograms showing propidium iodide fluorescence in MommeD7 ${ }^{+/+}$(left) and MommeD7-/+ (right) mice. In each case 10,000 reticulocytes were counted. Red blood cells (RBC), reticulocytes (RET) and nucleated cells (NC) are shown. (e) Histogram showing relative levels of GFP fluorescence in red blood cells and reticulocytes. Averages were calculated from three wild-type and three heterozygous mice. ${ }^{* *} p<0.005$; error bars represent SEM.

\section{Mapping}

We have mapped the mutations in all ten cases to relatively small regions of the genome (Table 4). The mapping of MommeD1-D6 has been documented [10]. Here we report the mapping of MommeD7-10. MommeD7 maps to a $0.25 \mathrm{Mb}$ region on chromosome 7 between markers D7Mit220 and rs13479441 (using 134 phenotypically mutant and 135 phenotypically wild-type mice). This region contains 10 genes. MommeD 8 maps to a $4 \mathrm{Mb}$ region on chromosome 4 between markers rs6337795 and D4Mit279 (using 234 phenotypically mutant and 177 phenotypically wild-type mice). This region contains 54 genes. MommeD9 maps to a $3 \mathrm{Mb}$ region on chromosome 7 between markers rs31712695 and rs6193818 (using 103 phenotypically mutant and 127 phenotypically wild-type mice). This region contains 80 genes. MommeD1O maps to a $4 \mathrm{Mb}$ region between markers D5Mit165 and rs13478547 on chromosome 5 . Twenty-four phenotypically homozygous and 312 phenotypically non-homozygous (heterozygous and wild-type mice combined) were used (see Materials and methods). These data show that each of the ten MommeD mutations maps to a unique region of the genome.

\section{MommeD5 has a mutation in Histone deacetylase I}

MommeD5 was localized to a $1.42 \mathrm{Mb}$ region on chromosome 4 flanked by the markers rs27486641 and rs27541967 [10] (Table 4). This interval contains 46 genes and Hdac1 was chosen as the best candidate because of its known role in chromatin modification. Sequencing of all exons, including exon/ intron boundaries, from two heterozygous and two wild-type individuals revealed a 7 bp deletion (GAAGCCA) in exon 13 in the mutants (Figure 5a). This mutation was subsequently verified in over 100 mice classified as mutants by GFP expression profiling. The chance of a second point mutation occurring in a functional region in a linked interval of this size is extremely small. Using the estimated mutation rate following ENU mutagenesis of 1 in $1.82 \mathrm{Mb}[15,16]$, the probability of such an event can be calculated $[15,17]$. This website takes into account the amount of coding sequence in a given linked interval. In this case, the probability of a second point mutation occurring in the coding region is $p<0.0005$.

The mutation produces a frameshift, resulting in changes to the last 12 amino acids, and an additional 25 amino acids. Protein modeling predictions based on human HDAC8, for which the crystal structure has been solved [18,19], suggest that the carboxyl terminus of Hdac1 is relatively unstructured and so the mutation is unlikely to affect the stability of the protein (J Matthews, personal communication). An antibody that recognizes the carboxyl terminus of Hdac1 showed a 50\% reduction of binding in $10.5 \mathrm{dpc}$ MommeD $5^{-/+}$embryos, and negligible binding in MommeD5/-- embryos (Figure 5b), confirming that this region of the protein has been altered in the MommeD5 line. An antibody that recognizes the amino terminus of Hdac1 showed that the levels of the protein are not altered between mutant and wild-type mice (Figure 5b). Lysine 476 at the carboxyl terminus has been shown to be sumoylated and important for enzymatic function of the wildtype protein [20] and the absence of this amino acid in the MommeD5 mutant protein is likely to impair function. A knockout of Hdac1 has been reported and the homozygous embryos died around 9.5 dpc [21], similar to the time of death 
Table 4

Summary of MommeD screen for epigenetic modifiers

\begin{tabular}{|c|c|c|c|c|}
\hline Name & Suppressor or enhancer of variegation & Mapping (Mb) & Mutated gene, or number of genes in interval & Homozygous lethality \\
\hline MommeDI & Suppressor & Chr 17 (2.4) & $\mathrm{SmcHDI}{ }^{*}$ & Females only EIO \\
\hline MommeD2 & Suppressor & Chr $9(1.4)$ & Dnmt $I^{\dagger}$ & E9 \\
\hline MommeD3 & Suppressor & Chr II (6) & 123 & E9-EI4 \\
\hline MommeD4 & Enhancer & Chr 8 (2.5) & Smarca5t & EI7 \\
\hline MommeD5 & Enhancer & Chr 4 (I.4) & Hdacl & E8-E9 \\
\hline MommeD6 & Suppressor & Chr I4 (2.5) & 32 & E8-E9 \\
\hline MommeD7 & Suppressor & Chr $7(0.25)$ & 10 & PI-P7 \\
\hline MommeD8 & Enhancer & Chr $4(4)$ & 54 & Most PI-P2I \\
\hline MommeD9 & Enhancer & Chr 7 (4) & 80 & E9 \\
\hline MommeDIO & Enhancer & Chr 5 (4) & $B a z l b$ & Most PI-P2I \\
\hline
\end{tabular}

All mapping data are current for Ensembl build 37.I, release 49. *Reported in [II]. tReported in [12].

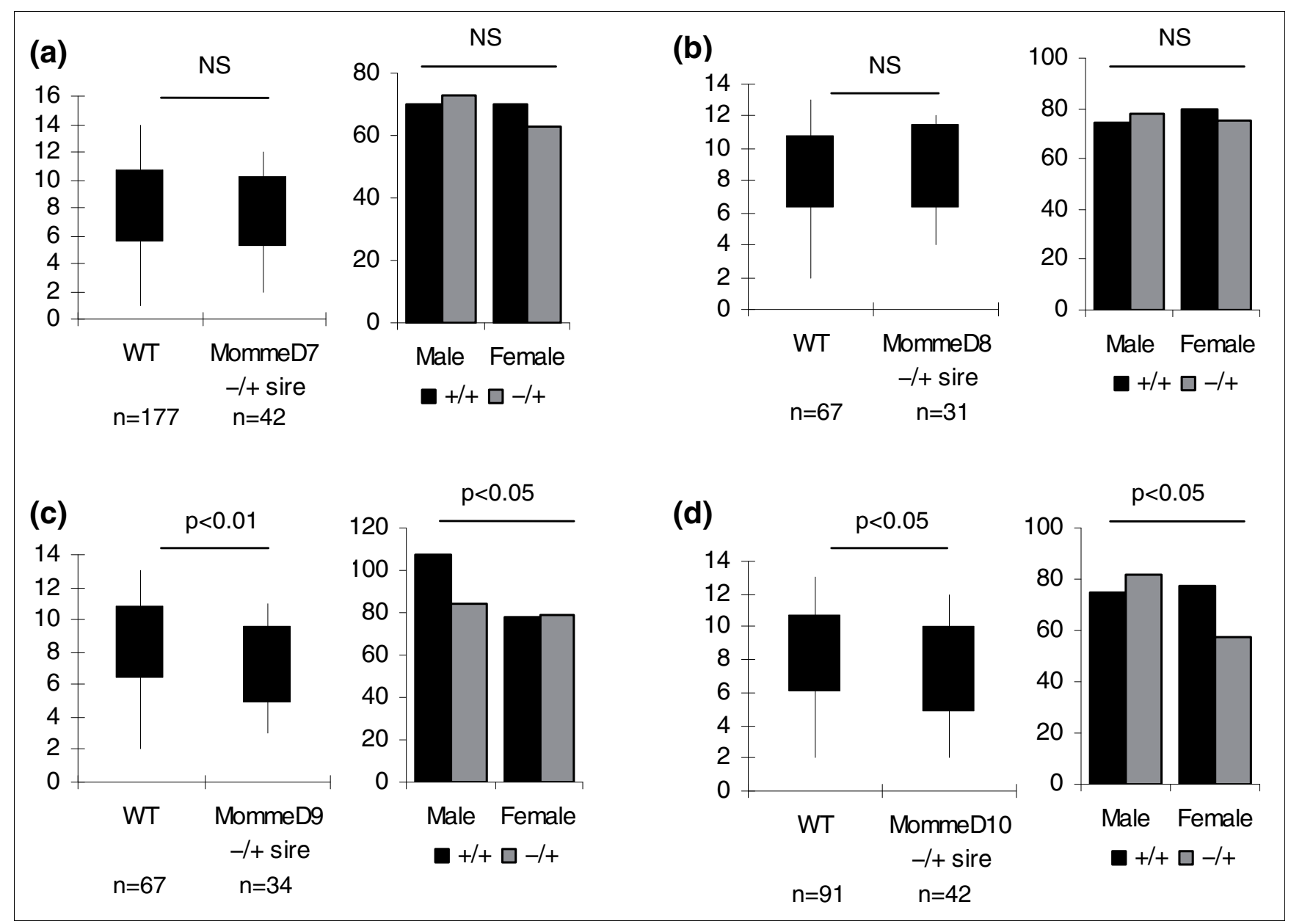

Figure 4

Genotypes and sex of offspring, and litter size following paternal transmission of MommeD7-DI0. (a) MommeD7, (b) MommeD8, (c) MommeD9, and (d) MommeDIO show the numbers of male and female offspring of wild-type (WT; black) and heterozygous (grey) genotype produced following a cross between a male heterozygous Momme and a wild-type female. MommeD9 and MommeD 10 both show a trend towards transmission ratio distortion and a significant reduction in litter size compared to wild-type crosses. $\mathrm{n}$ represents the number of litters produced. 


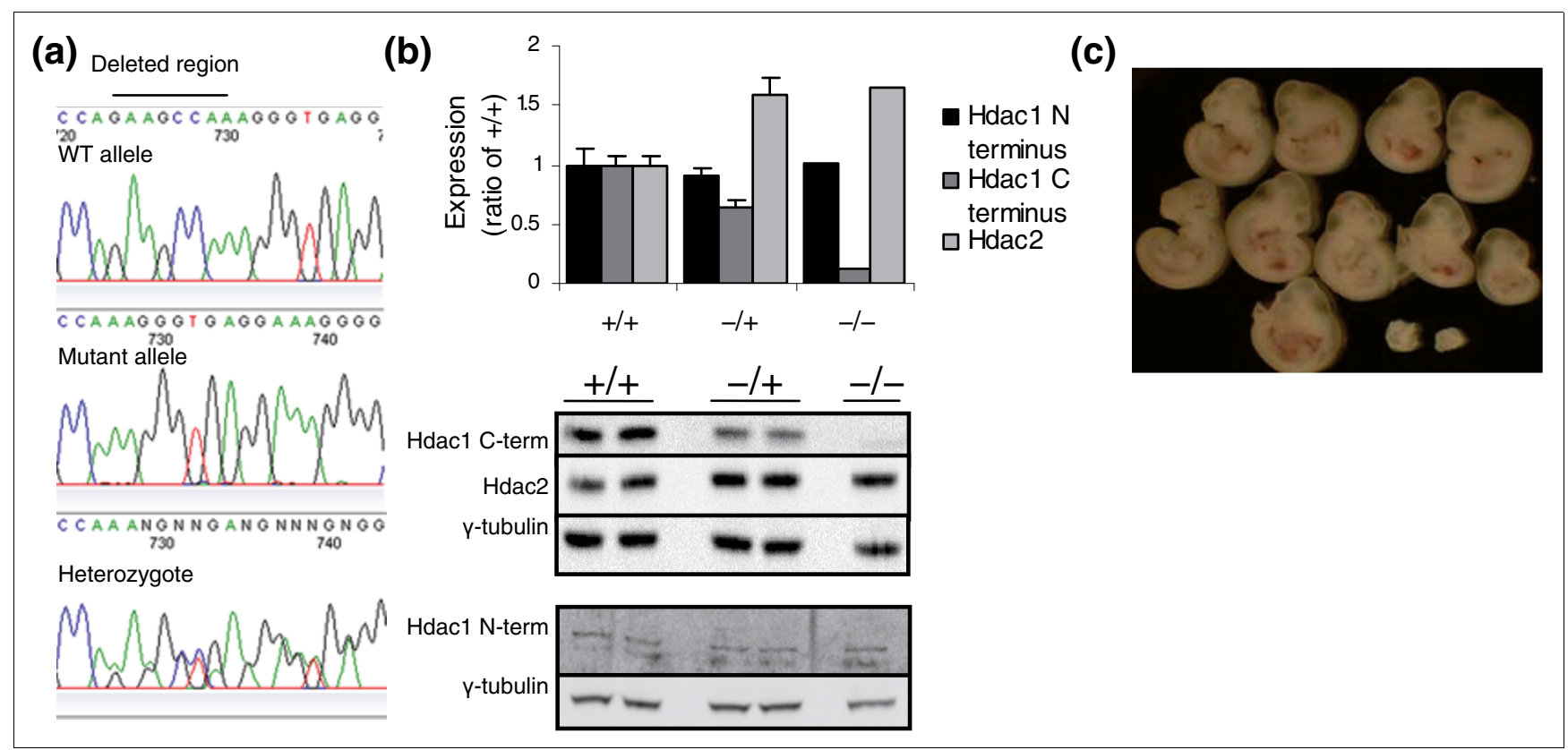

\section{Figure 5}

A mutation in Hdacl correlates with the MommeD5-/- phenotype. (a) A 7 bp deletion was detected in exon I 3 of Hdacl. Representative chromatograms from the wild-type (WT) allele, the mutant allele, and one heterozygote are shown. This deletion alters the reading frame, changing the last 12 amino acids and adding 25 extra amino acids. (b) Whole-cell lysates from six individual $10.5 \mathrm{dpc} \mathrm{MommeD}^{+/+}$and MommeD5-/+ embryos, and six pooled MommeD5 ${ }^{-/}$embryos were probed with antibodies to the Hdacl carboxyl terminus (top panel), Hdac2 (top panel) and Hdacl amino terminus (bottom panel). Anti- $\gamma$-tubulin was used as a loading control in each case. Quantification of the Hdacl carboxyl terminus relative to $\gamma$-tubulin shows negligible binding in MommeD5 ${ }^{-/-}$mice. Quantification of Hdac2 levels relative to $\gamma$-tubulin shows increased Hdac2 in MommeD5-/+ and MommeD5-/embryos. Quantification of $\mathrm{Hdacl}$ amino terminus relative to $\gamma$-tubulin shows equal levels of $\mathrm{Hdacl}$ in all mice. A peptide blocking experiment was performed to confirm band identity. A representative western blot is shown. Error bars represent SEM. (c) A representative litter from a MommeD5-/+ intercross at $10.5 \mathrm{dpc}$. MommeD5 ${ }^{-/-}$embryos (bottom right) are always dramatically smaller than MommeD5 ${ }^{+/+}$and MommeD5 $^{-/+}$littermates.

observed in MommeD5-/- embryos (Figure 5c). Together, these results argue that the mutation in Hdac1 is causative of the MommeD5 phenotype. Consistent with this, the level of Hdac2 increased in both MommeD5 $5^{-/+}$and MommeD5-/embryos, as predicted from the reports of compensatory upregulation of Hdac2 in embryonic stem cells null for Hdac1 [21]. Indeed, this upregulation may explain why MommeD5 was identified as an enhancer, rather than a suppressor, of variegation. Loss of histone deacetylase function is generally associated with transcriptional activation, but exceptions to this have been reported and the upregulation of Hdac2 could explain these results [22].

\section{MommeDIO has a mutation in Bazlb}

MommeD1O was localized to a $4 \mathrm{Mb}$ region on chromosome 5 flanked by the markers D5Mit165 and rs32250347 (Table 4). Interestingly, this interval encompasses the region syntenic with the Williams Beuren syndrome (WBS) critical region in humans. WBS, also known as Williams syndrome, is an autosomal dominant disorder affecting approximately 1 in 10,000 individuals. The classic presentation of WBS includes a characteristic craniofacial dysmorphology (elfin face), supravalvular aortic stenosis, multiple peripheral pulmonary arterial stenoses, statural deficiency, infantile hypocalcaemia and a distinct cognitive profile with mild mental retardation. The linked interval for MommeD1o contains 52 genes and Baz1b was chosen as the best candidate because it contains a bromodomain (a domain commonly associated with chromatin remodelers) and has recently been shown to form at least two chromatin remodeling complexes and associate with replication foci and promoters [23-25]. Sequencing of all exons, including exon/intron boundaries, from two homozygous, one heterozygous and one wild-type individual revealed one point mutation ( $\mathrm{T} \rightarrow \mathrm{G}$ transversion) in exon 7 in the mutants (Figure 6a). This mutation was subsequently verified in over 100 mice classified as mutants by GFP expression profile. The mutation results in a non-conservative amino acid change, L733R, in a highly conserved region of the protein (Figure 6b). Western blot analysis showed reduced levels of Baz1b protein in both embryonic and adult $\mathrm{MommeD}^{-/ /}$tissue, with $\mathrm{MommeD1O}^{-/+}$tissue showing intermediate levels (Figure $6 \mathrm{c}$ and data not shown), suggesting that the mutant protein is much less stable than its wild-type counterpart. Quantitative real-time PCR performed on cDNA from 14.5 dpc embryos showed all three genotypes have similar levels of mRNA (Figure 6d). 


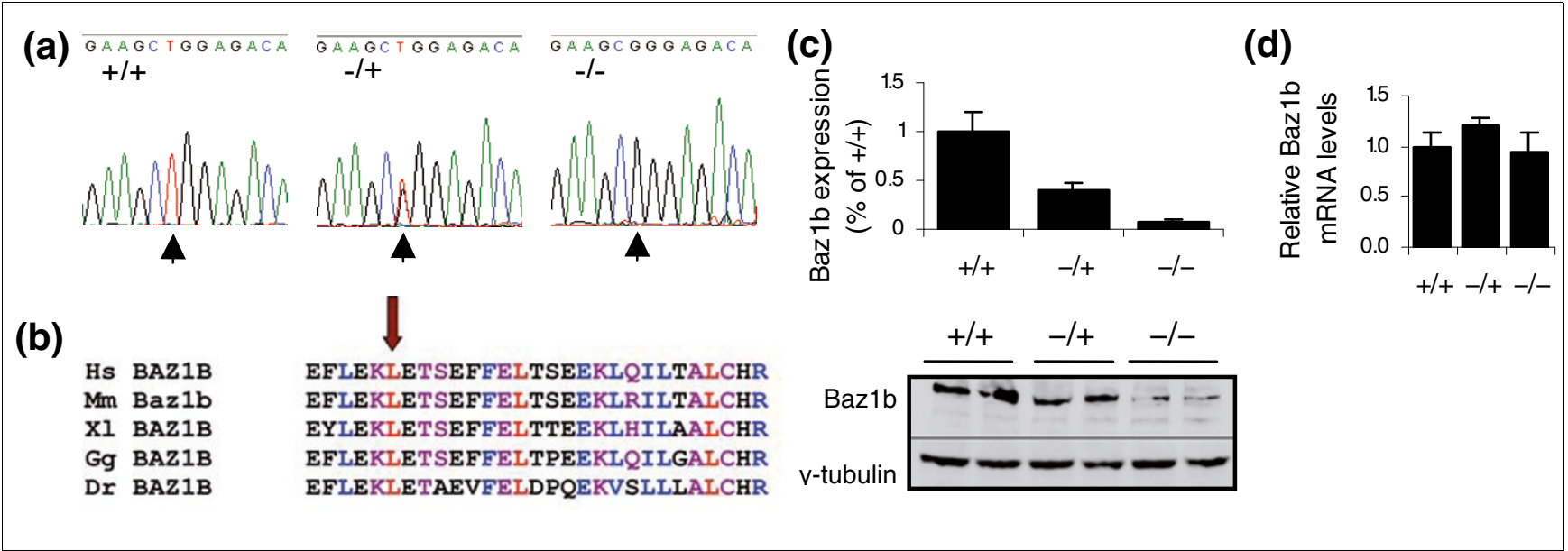

\section{Figure 6}

A point mutation in Bazlb correlates with the MommeD I0-/- phenotype. (a) A single base-pair mutation was detected in exon 7 of Baz $/ b$. Representative chromatograms from one wild type, one heterozygote and one homozygote are shown. This results in a non-conservative amino acid change, L733R. (b) The MommeDI 0 point mutation (arrow) modifies an amino acid highly conserved across species (Hs, Homo sapiens; Mm, Mus musculus; $\mathrm{XI}$, Xenopus laevis; Gg, Gallus gallus; Dr, Danio rerio). (c) Whole-cell lysates from four $14.5 \mathrm{dpc}$ heads of each genotype were probed with anti-Bazlb. Anti$\gamma$-tubulin was used as a loading control. Quantification of Bazlb relative to $\gamma$-tubulin shows negligible levels of Bazlb in MommeD/0-/- mice. A representative western blot is shown. (d) Expression analysis by quantitative real-time RT-PCR was performed on mRNA prepared from I4.5 dpc heads. Levels of Baz l b mRNA were not affected by the mutation. Analysis was performed on three individuals of each genotype and normalized to two housekeeping genes. Error bars in all graphs represent SEM.

\section{Effects of MommeD5 and MommeDIO on DNA methylation at the transgene locus}

Transgene silencing can be associated with changes in both DNA methylation [26,27] and chromatin accessibility [28]. This particular transgene promoter consists of a GC-rich segment of the human alpha-globin promoter, which we were unable to analyze by bisulfite sequencing because the cloned bisulfite converted fragment was refractory to growth in bacteria. The transgene also contains the HS-40 enhancer, which is known to regulate the locus in humans [29]. We analyzed the methylation state at this region by bisulfite sequencing. As predicted from the variegated nature of the transgene expression, the methylation pattern differed from clone to clone in all cases (data not shown). The percentage of methylated CpGs in the HS-40 element was approximately 55\% (averaged across all clones) in spleen from 4-week-old wild-type FVB/NJ mice (Figure 7a). Samples from MommeD5-/+, $\mathrm{MommeD}^{-/+}$, and MommeD1O-/- mice showed similar levels of CpG methylation (52\%, 47\%, 59\% respectively; Figure 7a). Mice heterozygous for a null allele of Dnmt3b, which showed an increase in expression of the GFP transgene from $37 \pm 3 \%$ in the wild-type mice to $55.5 \pm 2.5 \%$ in the Dnmt $3 b^{+/}$ - mice (in both cases mice were hemizygous for the transgene; see Materials and methods), showed a decrease in CpG methylation at the HS-40 element (31\%; Figure $7 \mathrm{~b})$ compared to that seen in the wild-type $\mathrm{C}_{57} \mathrm{BL} / 6 \mathrm{~J}$ mouse strain (60\%; Figure $7 \mathrm{~b}$ ). These data suggest that MommeD5 and MommeD1O mutants alter the expression of the transgene by changing the chromatin state rather than by altering DNA methylation levels. This is consistent with the fact that both genes encode proteins involved in histone modification and chromatin remodeling [21,23-25,30-33]. Modifiers identified in this screen include DNA methyltransferases, chromatin remodelers and genes involved in histone modification, all of which have wide-ranging effects in the genome, making it difficult to unravel direct and indirect effects at any particular locus.

\section{Craniofacial analysis of MommeDIO mice}

Surviving MommeD1o homozygotes were significantly smaller than littermates at weaning (Student's $t$-test, $p<$ o.0001; Figure 8a). A similar size differential was evident in utero at $18.5 \mathrm{dpc}$ (Student's $t$-test, $p<0.01$ ), indicating that this is not simply due to poor post-natal feeding (Figure 8a). MommeD1o homozygotes also appeared to have widened, bulbous foreheads and shortened snouts (Figure 8b). To examine the craniofacial phenotype more accurately, three heads from 4-week-old male mice of each genotype (MommeD1O-/-, MommeD1O ${ }^{-/+}$and $\mathrm{MommeD}^{+/+}$) were subjected to micro-computed tomography. Heads from one 4-week-old female of each genotype were also examined at this level. They followed the same trend as males. Visual inspection of the three-dimensional reconstructions confirmed the original observation that homozygote's skulls were more bulbous and showed a flattening of the nasal bone and upward curvature of the nasal tip (Figure 8c).

Twenty cranial landmarks and nine mandibular landmarks were located on each skull using approximately 70 micron resolution datasets and inter-landmark measurements were compared (Figure 8d and Additional data file 1). Statistical analyses were carried out using the data collected from males only. Homozygote skulls were significantly different to wild 


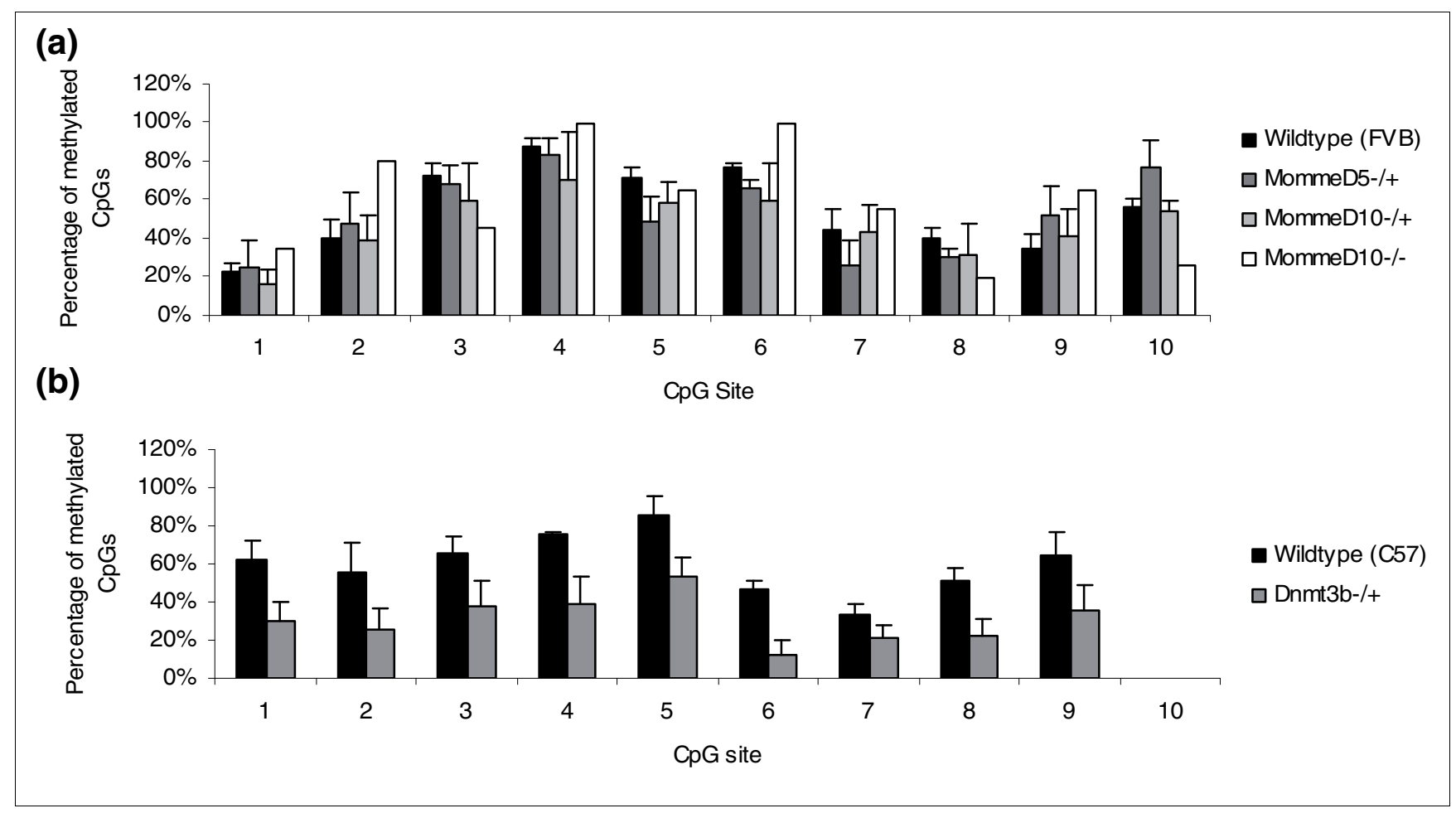

Figure 7

Bisulfite analysis of the HS-40 enhancer element. (a) DNA was extracted from the spleen of 4-week-old wild-type (FVB/NJ; three mice), MommeD5 ${ }^{-1+}$ (three mice), MommeD I $0^{-1+}$ (three mice) mice and from the tail of MommeD I0-/- mice (two mice). After bisulfite sequencing, the percentage of $C_{P} G$ methylation at each $C_{P G}$ site in the cloned region was analyzed, and averaged across individuals of the same genotype. None are significantly different from the wild-type mouse. Error bars represent SEM. (b) DNA was extracted from the tail of 4-week-old wild-type (C57BL/6; four mice) and Dnmt $3 b^{+/-}$(four mice) mice. After bisulfite sequencing, the percentage of $C_{p} G$ methylation at each $C_{p} G$ site in the cloned region was analyzed, and averaged across individuals of the same genotype. The $D n m t 3 b^{+/-}$mice have significantly $(p<0.001)$ less methylation than the wild-type mice. Error bars represent SEM.

type (Student's $t$-test, length:height ratio, $p<0.01$ width:height ratio, $p<0.01$; length:width ratio, $p<0.05$ ), confirming the bulbous appearance of the skulls on the reconstructed images. Much of this difference could be attributed to reduction of the parietal and nasal bones (both $>12.5 \%$ shorter in homozygotes compared to an overall mean length and width reduction of approximately $9 \%$ ). The reduced parietal bone length and the reduction and upward angulation of the nasal bones in these mice (Figure 8c, d) are reminiscent of the decrease in the volume of the parieto-occipital region and the appearance of the nose in WBS patients [34,35]. Heterozygotes also had a decreased cranium width:height ratio (Student's $t$-test, $p<0.05$ ) and decreased length:height ratio (Student's $t$-test, $p<0.05$ ) compared to wild-type skulls. Of note, heterozygotes showed a reduction in palatine bone width of similar magnitude to that seen in homozygotes, suggesting a greater sensitivity of some parts of the skull to decreased Baz1b protein levels. Measurements of the lower jaw revealed relative mandibular hypoplasia in homozygotes that was most pronounced in the posterior region (approximately 20\% reduction), encompassing the condyle, angle and coronoid processes (Figure 8d and Additional data file 1). The posterior aspects of the mandibles of heterozygotes were also reduced in size when compared to wild-type mandibles, albeit to a lesser degree than in the homozygotes.

\section{Expression of Bazlb during mouse embryogenesis}

It has previously been shown that Baz1b is expressed in the mouse embryo from around $9.5 \mathrm{dpc}$ and whole mount in situ at $11.5 \mathrm{dpc}$ showed expression in limb buds, tail and brain [24]. We have gone on to characterize the expression of Baz1b in more detail, and show that at $8.25 \mathrm{dpc}$ Baz1b is expressed in the headfolds, the caudal tail bud region and the presumptive hindbrain in a pattern reminiscent of rhombomere staining (Figure 9a, f). From 9.5 dpc expression is evident in the somites and in the forelimb bud as it emerges from the lateral plate mesoderm (Figure 9b). Diffuse mesenchymal expression in both the forelimb and hindlimb continues until 12.5 $\mathrm{dpc}$ when it is restricted to the interdigital mesenchyme (data not shown).

In the facial primordia, Baz1b is expressed in branchial arch 1 as it first emerges (Figure 9g), and continues later in development in both the maxillary and mandibular components of branchial arch 1 and branchial arch 2 (Figure 9c-e, h, i). Expression in the branchial arches is primarily mesenchymal, 


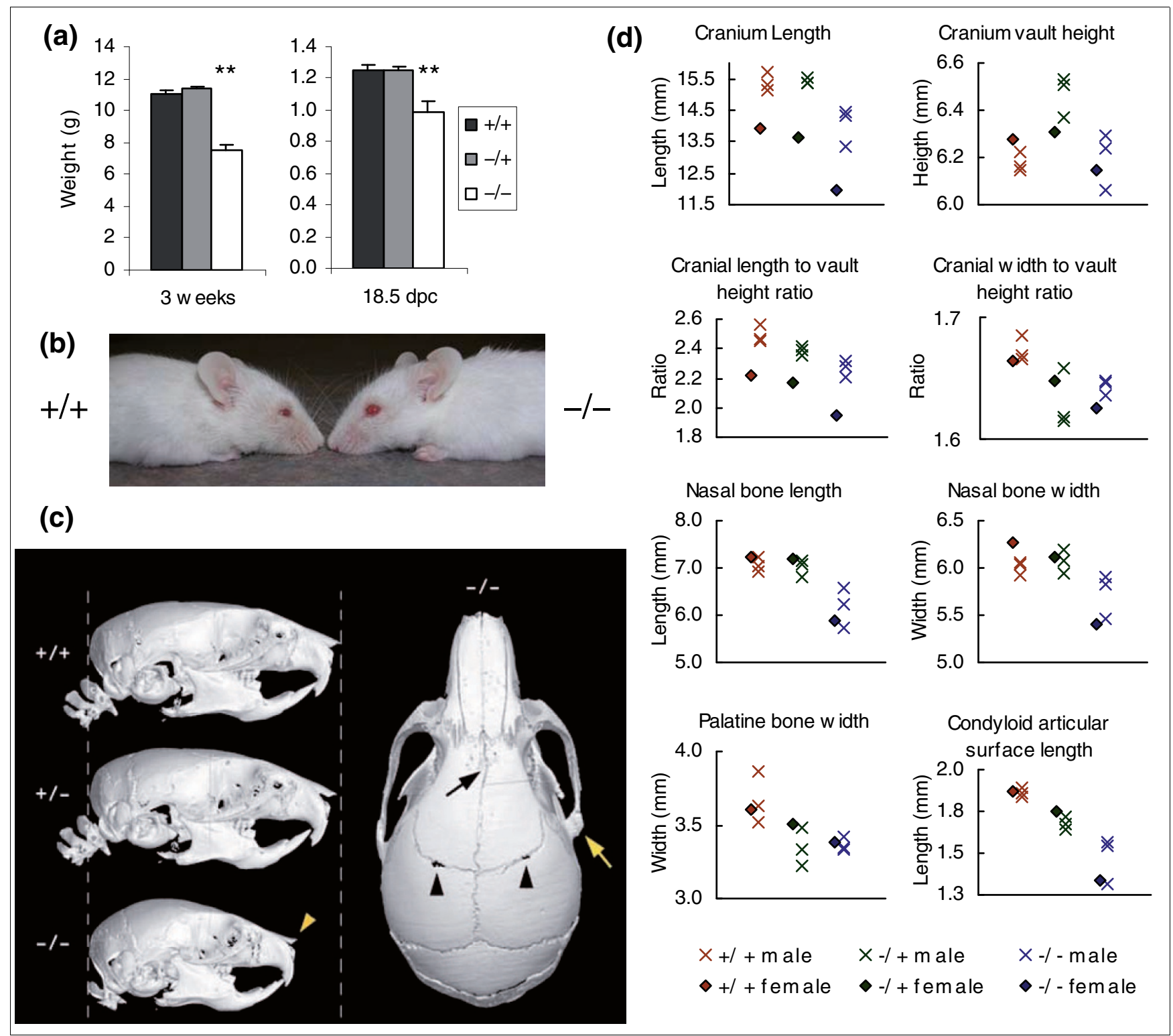

Figure 8

MommeDI $0^{-/-}$mice are smaller than their littermates and display craniofacial abnormalities. (a) Body weight was measured for 46

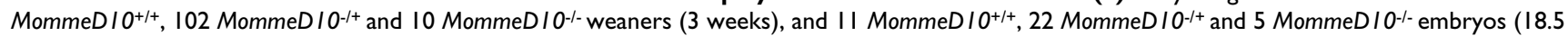
$\mathrm{dpc}$ ). Histograms show mean and SEM. (b) Craniofacial abnormalities in adult MommeD I $0^{-1-}$ mice. MommeD I $0^{-/-}$mice display shorter snouts than age and sex-matched wild-type littermates. (c) Three-dimensional reconstruction of skull microCT data from 4-week-old male mice reveals distinct anomalies in homozygous BazIB mice. Left side: lateral views show the overall size and shape of heterozygous skulls is similar to that of wild-type skulls, whereas skulls of homozygotes were around $20 \%$ shorter. Homozygous skulls showed variable anomalies, but consistently had a bulbous appearance, and a short, flattened, or upwardly angulated nasal bone (yellow arrowhead). Slight angulation of the nasal bones was also noted in one heterozygote. Right side: dorsal view of the homozygote skull shown on the left side showing the abnormal shape and more rostral connection of the zygomatic process with the squamosal bone (yellow arrow), skewing of the midline frontal bone suture (black arrow) and minor bilateral anomalies of the frontal:parietal suture (black arrowheads). (d) Twenty cranial landmarks and nine mandibular landmarks (based on those of Richtsmeier [49]) were located on each of nine skulls and inter-landmark measurements recorded. The mean value of each measurement, including analysis of cranium height:width and cranium length:height ratios, was compared between homozygous, heterozygous and wild-type animals. 


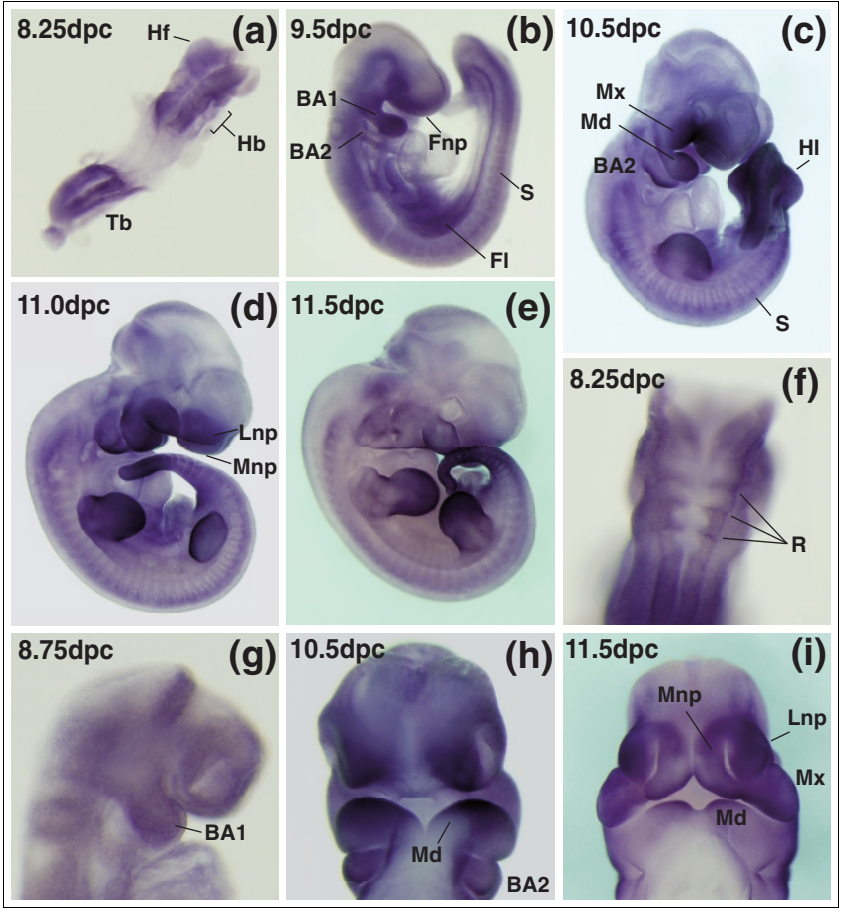

\section{Figure 9}

Whole mount in situ hybridization analysis in mouse embryos shows expression in the developing facial prominences. Embryos at a range of mid-gestational stages were hybridized with an antisense ribroprobe to the 3' untranslated region of Baz Ib. (a-e) Views of whole embryos from 8.25-1 I.5 dpc reveal strong expression in the mesenchyme of the maxillary and mandibular prominences, branchial arch 2 and the nasal processes. Other sites of $B a z l b$ expression include the presumptive hindbrain, headfolds and tailbud at $8.5 \mathrm{dpc}$, and the limb mesenchyme and somites at later stages. (f) Higher magnification dorsal view of presumptive hindbrain staining at $8.25 \mathrm{dpc}$. Close up images of facial prominence staining are shown at (g) $8.75 \mathrm{dpc}$, (h) $10.5 \mathrm{dpc}$, and (i) 11.5 $\mathrm{dpc}$. Images shown in $(\mathrm{h}, \mathrm{i})$ are ventral views following dissection of the head from the body of the embryo. BAI, branchial arch I; BA2, branchial arch 2; $\mathrm{Fl}$, forelimb; Fnp, frontonasal process; $\mathrm{Hb}$, hindbrain; $\mathrm{Hf}$, headfold; $\mathrm{HI}$, hindlimb; Lnp, lateral nasal prominence; Md, mandibular prominence; Mnp, medial nasal prominence; $M x$, maxillary prominence; $R$, rhombomere; S, somite; Tb, tailbud.

and is enriched in the rostral distal margin of the mandible, and the caudal distal margin of branchial arch 2 (Figure 9c-e, h). Baz1b is also expressed in the frontonasal process (Figure 9b) and later in the mesenchyme of both the medial and lateral nasal prominences as they elevate to surround the nasal pits (Figure 9c, d, h, i). Baz1b is expressed strongly in all the major facial primordia from early in embryogenesis.

\section{A possible role for $B a z I b$ in Williams syndrome}

Overall, the skull shape in mutant animals is reminiscent of the head shape seen in WBS, including a small upturned nose with flat nasal bridge, micrognathia (or mandibular hypoplasia), malocclusion, bi-temporal narrowing and prominent forehead [34]. WBS is known to be associated with a hemizygous deletion of approximately 28 genes in humans, but which of these genes are responsible for the craniofacial phenotype remains controversial. People with atypical deletions, and varying degrees of craniofacial abnormalities, implicate both proximal and distal ends of the deletion, suggesting that more than one gene is responsible [36-41]. Tassabehji and colleagues [42] reported craniofacial defects in a transgenic (c-myc; Tgf- $\alpha$ ) mouse line that harbored a chromosomal translocation fortuitously disrupting the Gtf2ird1 gene, the human orthologue of which resides in the WBS critical interval [43]. Mice homozygous for this transgene produced little Gtf2ird1 mRNA and exhibited craniofacial dysmorphism, suggesting a role for Gtf2ird1 in the craniofacial phenotype. Mice hemizygous for the transgene were indistinguishable from wild-type animals. Disruption of Gtf2ird1 in this mouse was associated with a $40 \mathrm{~kb}$ deletion at the site of integration, the addition of 5-10 tandem copies of a c-myc transgene, and translocation of the entire segment to chromosome 6 [43], providing opportunity for disruption to the expression of other genes, such as Baz1b, in the region. A targeted knockout of Gtf2ird1, produced by others, failed to find craniofacial dysmorphology or dental abnormalities [44]. We checked the sequence and expression of Gtf2ird1 in MommeD1O mutants and found no abnormalities (data not shown). The chance of a second point mutation occurring in a coding region in this linked interval is extremely small ( $p=$ 0.0008 , based on a mutation rate of 1 in $1.82 \mathrm{Mb}$ ) [15-17].

Our studies show that the chromatin remodeler Baz1b is expressed strongly in cranial neural crest-derived mesenchyme that drives facial morphogenesis and that homozygosity for a missense mutation in Baz1b produces an array of craniofacial features that are similar to those that characterize the typical WBS face. Significantly, some craniofacial features are also apparent in heterozygotes. These results suggest that reduction in Baz1b protein levels contributes to the elfin facies characteristic of WBS and that WBS is, at least in part, a chromatin-remodeling factor disease.

\section{Conclusion}

Extension of the screen has produced four new MommeDs, MommeD7-D1O, all of which behave in a semidominant fashion, as do the six previously reported [10]. We have now identified the underlying genes in five of the ten cases, two of which, Hdac1 and Baz1b, we report here. Both are already known to be involved in epigenetic processes, further validating the screen design. In the case of $B a z 1 b$ this is the first report of a mouse carrying a disrupted allele at this locus and we have shown a role for the protein in craniofacial development.

The screen, designed primarily to identify genes involved in the epigenetic gene silencing of foreign DNA, such as transgenes, has revealed the critical role that such genes play in embryonic and fetal development. It is interesting that at least half of the MommeDs are important during gastrulation. Furthermore, the identification of heterozygous effects sug- 
gests that a reduction in the amount of protein product of less than $50 \%$ has effects on the health of the mouse. One of the hallmarks of epigenetic gene silencing across all multicellular organisms from plants to humans is the stochastic nature by which they operate [45] and these studies re-emphasize the importance of probabilistic events during embryogenesis. We believe that this screen will provide new tools with which to study these processes.

\section{Materials and methods \\ Mouse strains}

Wild-type inbred $\mathrm{C}_{57 \mathrm{BL} / 6 J}$ and FVB/NJ mice were purchased from ARC Perth. Procedures were approved by the Animal Ethics Committee of the University of Sydney and the Animal Ethics Committee of the Queensland Institute of Medical Research. The ENU screen was carried out in the FVB/NJ inbred transgenic line as described previously [10]. All MommeD lines were maintained in this strain unless stated otherwise. Most crosses between MommeD individuals were performed on individuals three generations or more removed from the MommeD progenitor. A total of $1,000 \mathrm{G} 1$ offspring were screened at 3 weeks of age, from which ten heritable dominant mutations were identified.

The Dnmt3b null mice were a kind gift from En Li. They have been subsequently backcrossed for more than ten generations to the $\mathrm{C} 57 \mathrm{BL} / 6 \mathrm{~J}$ strain. GFP fluorescence in Dnmt $3 b^{+/-}$mice was analyzed in the $\mathrm{F}_{1}$ offspring of crosses between $D n m t 3 b^{+/}$ - mice and the FVB/NJ transgenic line, and as such each mouse was hemizygous for the transgene.

\section{Flow cytometry}

GFP fluorescence in erythrocytes was analyzed by flow cytometry at weaning. A drop of blood was collected in Osmosol buffer (Lab Aids Pty Ltd, Narrabeen, NSW, Australia) and analyzed on a FACScan (Becton Dickinson, Franklin Lakes, NJ, USA) with excitation at 488 and $550 \mathrm{~nm}$. The $488 \mathrm{~nm}$ channel predominantly measures GFP fluorescence and the $550 \mathrm{~nm}$ channel measures autofluoresence. The data were analyzed using CELL QUEST software with a GFP-positive gate set to exclude $99.9 \%$ of wild-type erythrocytes. Mean fluorescence was calculated using cells within the positive gate. Histograms depict only the GFP fluorescence channel.

\section{Genome walking}

Genome walking was performed using the APAgene ${ }^{\mathrm{TM}}$ GOLD Genome Walking Kit (Bio S\&T Inc., Montreal, Quebec, Canada) following the manufacturer's instructions. Gene specific primers used were (5'-3'): WalkA CCATATTTTCACCATACACGACA; WalkB GAGACTTTCTCATCCCCAAAACT; WalkC CCCCAAAACTTGTACCCAAA.

\section{Linkage analysis}

For MommeD7, D8 and D9, FVB/C57 F1 heterozygous individuals were backcrossed to $\mathrm{C}_{57}$, and linkage analysis per- formed on their offspring. Phenotype was assigned based on GFP fluorescence profile. A panel of microsatellite markers that differ in size between FVB and $\mathrm{C}_{57}$ were used to localize the mutation to a small area of the genome. Mice wild type for the mutation should only have $\mathrm{C}_{57}$ chromosomes at the linked interval, while mice heterozygous for the mutation should carry both FVB and C57 chromosomes.

Linkage analysis in MommeD1O was carried out using an $\mathrm{FVB} / \mathrm{C} 57 \mathrm{~F} 1 \mathrm{MommeD}^{-/ /+}$intercross to produce $337 \mathrm{~F} 2$ individuals. $M o m m e D 1 O^{-/-}$mice were distinguished from their littermates by their dramatically reduced size at weaning and their reduced GFP expression profile. Recombination events allowed the linked region to be localized to a small genomic interval. MommeD1O-/- mice should only carry FVB chromosomes at the MommeD1o linked region, while the remaining mice should be either FVB/C57 or C57/C57.

\section{Reticulocyte counts}

Blood smears were made from blood taken from the tail tip of MommeD7 $7^{-/+}$and MommeD7 $7^{+/+}$mice, and stained with New methylene blue. Full blood analyses were done on the automated haematology analyzer Sysmex Xe-2100 (Sysmex Corporation, Woodlands Spectrum, Singapore).

\section{Reticulocyte analysis by FACS}

Nucleated cells and reticulocytes were separated from mature erythrocytes based on propidium iodide fluorescence levels. An RNase control was performed and the presumptive reticulocyte cell population could no longer be detected. Mean GFP fluorescence was determined for reticulocyte and mature erythrocyte cell populations. This was done essentially as described in [46]. Three adult MommeD $7^{/+}$and three wildtype littermate controls were used. Approximately $25 \mu \mathrm{l}$ of whole blood was collected from the tail in cold Osmosol buffer (Lab Aids Pty Ltd). Cells were fixed for $1 \mathrm{~h}$ at $4{ }^{\circ} \mathrm{C}$ in $1 \%$ paraformaldehyde in Osmosol and then washed once in cold Osmosol. Cells were then permeabilized by adding $-20^{\circ} \mathrm{C}$ ethanol to a cell pellet whilst vortexing, and incubated with rotation for $2.5 \mathrm{~h}$ at $4^{\circ} \mathrm{C}$. A $40 \mu \mathrm{g} / \mathrm{ml}$ solution of propidium iodide was added to pelleted cells and the cells were incubated at $37^{\circ} \mathrm{C}$ for 30 minutes. Analysis was performed on a FACScan (Becton Dickinson). The data were analyzed using CELL QUEST software.

\section{Genotyping assay}

Following identification of the MommeD1o point mutation, genotyping was carried out by AciI digestion of a PCR product of exon 7 of Baz1b (primers available on request). The AciI site is created by the MommeD1o point mutation. Following identification of the MommeD5 mutation, genotyping was carried out by PCR amplification across the deleted region, and gel electrophoresis using Ultra low-range agarose (BioRad, Hercules, CA, USA). 


\section{Protein analysis}

We prepared whole-cell lysates of $10.5 \mathrm{dpc}$ embryos (MommeD5) and $14.5 \mathrm{dpc}$ heads and adult spleen (MommeD1O) in 2-3× volume lysis buffer (0.05 M Tris, $7 \mathrm{M}$ urea, $150 \mathrm{mM} \mathrm{NaCl}$ ). Samples were incubated on ice for 20 minutes, sonicated, centrifuged, and the supernatant collected. Approximately $5 \mu \mathrm{g}$ (MommeD5) or $10 \mu \mathrm{g}$ (MommeD1O) of protein was separated by SDS-PAGE on a 4$12 \%$ gradient gel (Invitrogen, Carlsbad, CA, USA) and was analyzed with antibodies to the Hdac1 amino terminus (sc6299, Santa Cruz Biotechnology, Santa Cruz, CA, USA), Hdac1 carboxyl terminus (05-100, Millipore, Billerica, MA, USA), Hdac2 (05-814, Millipore), Baz1B (BL2067, Bethyl Laboratories, Montgomery, TX, USA) or $\gamma$-tubulin (T5192, Sigma-Aldrich, St Louis, MO, USA). Blots were quantified by normalizing the levels of Hdac1, Hdac2 or Baz1B in each lane to that of $\gamma$-tubulin. The normalized levels were then averaged across genotypes and the ratio to wild-type levels calculated. A peptide blocking experiment was carried out for the Hdac1 amino terminus using sc-6299P (Santa Cruz Biotechnology).

\section{RNA preparation and quantitative RT-PCR}

Total RNA was extracted from 14.5 dpc embryo heads using TRI reagent (Sigma-Aldrich). cDNA was synthesized from total RNA using SuperScriptII reverse transcriptase (Invitrogen) and random hexamer primers. Quantitative RT-PCR was performed with the Platinum SYBR Green qPCR SuperMix-UDG with primers designed to span exon/intron boundaries (available on request). All reactions were performed in triplicate and normalized to both GAPDH and ribosomal highly basic 23-kDa protein (Rpl13A) [47]. The reaction was run on a Corbett Research Rotor-Gene (Qiagen, Doncaster, Vic, Australia).

Bisulfite sequencing of the transgene HS-40 enhancer Bisulfite sequencing was carried out as described in [48]. Oligonucleotides to the bisulfite converted HS-40 enhancer were as follows (5'-3'): GFPbisF1 AAAATAAAATTTTTGGATTGTTATTATTATAA; GFPbisF2 ATATTTGTAATTTTAGTATTTTGGGAGGTT; and GFPbisR AATCTCTACTCACTACAAACTCCATCTC. Cycling conditions were as follows: $94^{\circ} \mathrm{C}$ for 2 minutes for 1 cycle; $94^{\circ} \mathrm{C}$ for $30 \mathrm{~s}$, $60^{\circ} \mathrm{C}$ for $30 \mathrm{~s}, 72^{\circ} \mathrm{C}$ for $45 \mathrm{~s}$ for 35 cycles; and $72^{\circ} \mathrm{C}$ for $6 \mathrm{~min}$ utes for 1 cycle.

\section{Micro-CT analysis}

Three heads of 4-week-old male mice of each genotype, and one head of female mice of each genotype (MommeD1O-/, $\mathrm{MommeD}^{-/+}$and $\mathrm{MommeD}^{-\mathrm{O}^{+/+}}$) were scanned at 8.7 micron resolution using a SkyScan 1076 micro-computed tomography unit and the data set reduced to approximately 17 microns for three-dimensional reconstruction of the serial slices (CT Analyser software V.1.6.1.1; SkyScan, Kontich, Belgium). Twenty cranial landmarks and nine mandibular landmarks (based on those of Richtsmeier [49]) were located on each of nine skulls and inter-landmark measurements recorded using DataViewer software (V.1.3.o.o; SkyScan). To verify accuracy of the measurements, any landmarks showing marked differences between genotype groups were re-located on a separate day and the measurement repeated. The mean value of each measurement was compared between homozygotes, heterozygotes and wild-type animals.

\section{RNA probes and whole-mount in situ hybridization}

DIG-labeled RNA probes were transcribed from linearized DNA templates and used in whole mount in situ hybridization analysis of wild-type FVB/NJ mouse embryos at a range of gestational stages. The probe was directed to $1.1 \mathrm{~kb}$ of the $3^{\prime}$ untranslated region of $B a z 1 b$. A sense probe was used in earlier experiments to confirm specificity of the antisense probe. Whole mount in situ hybridization was performed as previously described [50]. Embryo images were captured digitally using an Olympus SZX12 microscope with DP Controller software (Olympus Corporation).

\section{Abbreviations}

dpc: days post-coitus; ENU: N-ethyl-N-nitrosourea; FACS: fluorescence activated cell sorting; GFP: green fluorescent protein; Momme: Modifier of murine metastable epialle; SEM: standard error of the mean; WBS: Williams Beuren syndrome.

\section{Authors' contributions}

AA carried out the genetic and molecular studies for MommeD2 and MommeD1o, the FACS analysis on whole blood for MommeD7 and co-wrote the manuscript. DKM carried out the genetic and molecular studies for MommeD5. NCW carried out the genetic analysis on MommeD7, MommeD8, and MommeD9 and determined the transgene integration site. TJB carried out genetic studies on MommeD6 and initial analysis on MommeD9. NKV performed ENU mutagenesis and carried out genetic studies on MommeD4 and initial analysis on MommeD7. LLC carried out the craniofacial analysis. NCB and CW performed and interpreted the whole mount in situ hybridizations. MEB performed ENU mutagenesis and carried out genetic studies on MommeD1 and MommeD3. SJW and GJA performed the reticulocyte staining. TCC carried out the craniofacial analysis and interpretation. EW conceived the study, and project design, coordinated the project and together with other coauthors interpreted results and wrote the manuscript. All authors read and approved the final manuscript.

\section{Additional data files}

The following additional data file is available with the online version of this paper. Additional data file 1 shows more craniofacial measurements between $\mathrm{MommeD}_{1} \mathrm{O}^{+/+}$, $\mathrm{MommeD}^{-/+}$and $\mathrm{MommeD}^{-/-} \mathrm{O}^{-/}$mice. 


\section{Acknowledgements}

The authors would like to thank Belinda Washbourne and Dr Peter Self at Adelaide Microscopy for assistance with the microcomputed tomography scans and logistics, and Dr J Matthews at The University of Sydney for assistance with protein modeling. This study was supported by NHMRC grants to EW. AA, DKM, NCW, TB and NCB were supported by Australian Postgraduate Awards. NV was supported by an International Postgraduate Award (University of Sydney). CW and GJA are NHMRC Senior Research Fellows. EW was supported by a fellowship from the Queensland Institute of Medical Research.

\section{References}

I. Henikoff S: Position-effect variegation after $\mathbf{6 0}$ years. Trends Genet 1990, 6:422-426.

2. Spofford JB: Parental control of position-effect variegation. II. Effect of sex of parent contributing white-mottled rearrangement in Drosophila melanogaster. Genetics |96|, 46:115I-II67.

3. Wolff GL: Influence of maternal phenotype on metabolic differentiation of agouti locus mutants in the mouse. Genetics 1978, 88:529-539.

4. Rakyan VK, Blewitt ME, Druker R, Preis JI, Whitelaw E: Metastable epialleles in mammals. Trends Genet 2002, 18:348-35I.

5. Waterland RA, Jirtle RL: Transposable elements: targets for early nutritional effects on epigenetic gene regulation. Mol Cell Biol 2003, 23:5293-5300.

6. Morgan HD, Sutherland HG, Martin DI, Whitelaw E: Epigenetic inheritance at the agouti locus in the mouse. Nat Genet 1999, 23:3|4-3|8.

7. Reuter G, Spierer P: Position effect variegation and chromatin proteins. Bioessays 1992, I 4:605-6I2.

8. Schotta G, Ebert A, Dorn R, Reuter G: Position-effect variegation and the genetic dissection of chromatin regulation in Drosophila. Semin Cell Dev Biol 2003, 14:67-75.

9. Dorn $R$, Krauss $V$, Reuter $G$, Saumweber $H$ : The enhancer of position-effect variegation of Drosophila, E(var)3-93D, codes for a chromatin protein containing a conserved domain common to several transcriptional regulators. Proc Natl Acad Sci USA 1993, 90: I I376-II380.

10. Blewitt ME, Vickaryous NK, Hemley SJ, Ashe A, Bruxner TJ, Preis JI, Arkell R, Whitelaw E: An N-ethyl-N-nitrosourea screen for genes involved in variegation in the mouse. Proc Natl Acad Sci USA 2005, 102:7629-7634.

II. Blewitt ME, Gendrel AV, Pang Z, Sparrow DB, Whitelaw N, Craig JM, Apedaile A, Hilton DJ, Dunwoodie SL, Brockdorff N, Kay GF, Whitelaw E: SmcHDI, containing a structural-maintenanceof-chromosomes hinge domain, has a critical role in $\mathbf{X}$ inactivation. Nat Genet 2008, 40:663-669.

12. Chong S, Vickaryous N, Ashe A, Zamudio N, Youngson N, Hemley S, Stopka T, Skoultchi A, Matthews J, Scott HS, de Kretser D, O'Bryan $M$, Blewitt $M$, Whitelaw E: Modifiers of epigenetic reprogramming show paternal effects in the mouse. Nat Genet 2007, 39:614-622.

13. Garrick D, Fiering S, Martin DI, Whitelaw E: Repeat-induced gene silencing in mammals. Nat Genet 1998, 18:56-59.

14. Preis JI, Downes M, Oates NA, Rasko JE, Whitelaw E: Sensitive flow cytometric analysis reveals a novel type of parent-of-origin effect in the mouse genome. Curr Biol 2003, I 3:955-959.

15. Keays DA, Clark TG, Campbell TG, Broxholme J, Valdar W: Estimating the number of coding mutations in genotypic and phenotypic driven $\mathrm{N}$-ethyl-N-nitrosourea (ENU) screens: revisited. Mamm Genome 2007, 18:123-124.

16. Keays DA, Clark TG, Flint J: Estimating the number of coding mutations in genotypic- and phenotypic-driven $\mathrm{N}$-ethyl- $\mathrm{N}$ nitrosourea (ENU) screens. Mamm Genome 2006, 17:230-238.

17. Estimating the probability of ENU-induced coding mutations [http://www.well.ox.ac.uk/enuMutRat]

18. Somoza JR, Skene RJ, Katz BA, Mol C, Ho JD, Jennings AJ, Luong C, Arvai A, Buggy JJ, Chi E, Tang J, Sang BC, Verner E, Wynands R, Leahy EM, Dougan DR, Snell G, Navre M, Knuth MW, Swanson RV, McRee DE, Tari LW: Structural snapshots of human HDAC8 provide insights into the class I histone deacetylases. Structure 2004, 12:1325-1334

19. Vannini A, Volpari C, Filocamo G, Casavola EC, Brunetti M, Renzoni D, Chakravarty P, Paolini C, De Francesco R, Gallinari P, Steinkühler
C, Di Marco S: Crystal structure of a eukaryotic zinc-dependent histone deacetylase, human HDAC8, complexed with a hydroxamic acid inhibitor. Proc Natl Acad Sci USA 2004, I0I:I5064-I5069.

20. David G, Neptune MA, DePinho RA: SUMO-I modification of histone deacetylase I (HDACI) modulates its biological activities. J Biol Chem 2002, 277:23658-23663.

2I. Lagger G, O'Carroll D, Rembold M, Khier H, Tischler J, Weitzer G, Schuettengruber B, Hauser C, Brunmeir R, Jenuwein T, Seiser C: Essential function of histone deacetylase $I$ in proliferation control and CDK inhibitor repression. EMBO J 2002, 21:2672-268I.

22. Zupkovitz G, Tischler J, Posch M, Sadzak I, Ramsauer K, Egger G, Grausenburger R, Schweifer N, Chiocca S, Decker T, Seiser C: Negative and positive regulation of gene expression by mouse histone deacetylase I. Mol Cell Biol 2006, 26:7913-7928.

23. Bozhenok L, Wade PA, Varga-Weisz P: WSTF-ISWI chromatin remodeling complex targets heterochromatic replication foci. EMBO J 2002, 21:2231-224I.

24. Kitagawa $H$, Fujiki R, Yoshimura K, Mezaki $Y$, Uematsu $Y$, Matsui $D$, Ogawa S, Unno K, Okubo M, Tokita A, Nakagawa T, Ito T, Ishimi Y, Nagasawa H, Matsumoto T, Yanagisawa J, Kato S: The chromatinremodeling complex WINAC targets a nuclear receptor to promoters and is impaired in Williams syndrome. Cell 2003, I I 3:905-9|7.

25. Percipalle P, Fomproix N, Cavellan E, Voit R, Reimer G, Kruger T, Thyberg J, Scheer U, Grummt I, Farrants AK: The chromatin remodelling complex WSTF-SNF2h interacts with nuclear myosin I and has a role in RNA polymerase I transcription. EMBO Rep 2006, 7:525-530.

26. Allen ND, Norris ML, Surani MA: Epigenetic control of transgene expression and imprinting by genotype-specific modifiers. Cell 1990, 61:853-86I.

27. McGowan R, Campbell R, Peterson A, Sapienza C: Cellular mosaicism in the methylation and expression of hemizygous loci in the mouse. Genes Dev 1989, 3:1669-1676.

28. Garrick D, Sutherland H, Robertson G, Whitelaw E: Variegated expression of a globin transgene correlates with chromatin accessibility but not methylation status. Nucleic Acids Res 1996 , 24:4902-4909.

29. Vyas P, Vickers MA, Simmons DL, Ayyub H, Craddock CF, Higgs DR: Cis-acting sequences regulating expression of the human alpha-globin cluster lie within constitutively open chromatin. Cell 1992, 69:781-793.

30. Ahringer J: NuRD and SIN3 histone deacetylase complexes in development. Trends Genet 2000, 16:35I-356.

31. Taunton J, Hassig CA, Schreiber SL: A mammalian histone deacetylase related to the yeast transcriptional regulator Rpd3p. Science 1996, 272:408-4II.

32. Fujiki R, Kim MS, Sasaki Y, Yoshimura K, Kitagawa H, Kato S: Ligandinduced transrepression by VDR through association of WSTF with acetylated histones. EMBO J 2005, 24:388I-3894.

33. Poot RA, Bozhenok L, Berg DL van den, Hawkes N, Varga-Weisz PD Chromatin remodeling by WSTF-ISWI at the replication site: opening a window of opportunity for epigenetic inheritance? Cell Cycle 2005, 4:543-546.

34. Perez Jurado AL: Williams-Beuren syndrome: a model of recurrent genomic mutation. Horm Res 2003, 59(Suppl I): $106-113$.

35. Schmitt JE, Eliez S, Bellugi U, Reiss AL: Analysis of cerebral shape in Williams syndrome. Arch Neurol 200I, 58:283-287.

36. Botta A, Novelli G, Mari A, Novelli A, Sabani M, Korenberg J, Osborne LR, Digilio MC, Giannotti A, Dallapiccola B: Detection of an atypical $7 \mathrm{ql}$ I.23 deletion in Williams syndrome patients which does not include the STXIA and FZD3 genes. J Med Genet 1999, 36:478-480.

37. Duba HC, Doll A, Neyer M, Erdel M, Mann C, Hammerer I, Utermann $\mathrm{G}$, Grzeschik $\mathrm{KH}$ : The elastin gene is disrupted in a family with a balanced translocation $t(7 ; 16)(q \mid I .23 ; q / 3)$ associated with a variable expression of the Williams-Beuren syndrome. Eur J Hum Genet 2002, 10:35I-36I.

38. Edelmann L, Prosnitz A, Pardo S, Bhatt J, Cohen N, Lauriat T, Ouchanov L, González PJ, Manghi ER, Bondy P, Esquivel M, Monge S, Delgado MF, Splendore A, Francke U, Burton BK, Mclnnes LA: An atypical deletion of the Williams-Beuren syndrome interval implicates genes associated with defective visuospatial processing and autism. J Med Genet 2007, 44: I36-143.

39. Hirota H, Matsuoka R, Chen XN, Salandanan LS, Lincoln A, Rose FE, 
Sunahara M, Osawa M, Bellugi U, Korenberg JR: Williams syndrome deficits in visual spatial processing linked to GTF2IRDI and GTF2I on chromosome 7qII.23. Genet Med 2003, 5:3II-32I.

40. Howald C, Merla G, Digilio MC, Amenta S, Lyle R, Deutsch S, Choudhury U, Bottani A, Antonarakis SE, Fryssira H, Dallapiccola B, Reymond A: Two high throughput technologies to detect segmental aneuploidies identify new Williams-Beuren syndrome patients with atypical deletions. J Med Genet 2006, 43:266-273.

41. Osborne LR, Li M, Pober B, Chitayat D, Bodurtha J, Mandel A, Costa T, Grebe T, Cox S, Tsui LC, Scherer SW: A I.5 million-base pair inversion polymorphism in families with Williams-Beuren syndrome. Nat Genet 200I, 29:32I-325.

42. Tassabehji M, Hammond P, Karmiloff-Smith A, Thompson P, Thorgeirsson SS, Durkin ME, Popescu NC, Hutton T, Metcalfe K, Rucka A, Stewart H, Read AP, Maconochie M, Donnai D: GTF2IRDI in craniofacial development of humans and mice. Science 2005, 310:1 184-1187.

43. Durkin ME, Keck-Waggoner CL, Popescu NC, Thorgeirsson SS: Integration of a c-myc transgene results in disruption of the mouse Gtf2ird I gene, the homologue of the human GTF2IRDI gene hemizygously deleted in Williams-Beuren syndrome. Genomics 200I, 73:20-27.

44. Palmer SJ, Tay ES, Santucci N, Cuc Bach TT, Hook J, Lemckert FA, Jamieson RV, Gunnning PW, Hardeman EC: Expression of Gtf2ird I, the Williams syndrome-associated gene, during mouse development. Gene Expr Patterns 2007, 7:396-404.

45. Martin $\mathrm{DI}$, Whitelaw $\mathrm{E}$ : The vagaries of variegating transgenes. Bioessays 1996, I 8:919-923.

46. Schmid I, Sakamoto KM: Analysis of DNA content and green fluorescent protein expression. Curr Protoc Cytom 200I, Chapter 7:Unit 7.16.

47. Mogal A, Abdulkadir SA: Effects of histone deacetylase inhibitor (HDACi); Trichostatin-A (TSA) on the expression of housekeeping genes. Mol Cell Probes 2006, 20:8I-86.

48. Rakyan VK, Chong S, Champ ME, Cuthbert PC, Morgan HD, Luu $K V K$, Whitelaw $E$ : Transgenerational inheritance of epigenetic states at the murine Axin $\mathrm{Fu}$ allele occurs after maternal and paternal transmission. Proc Natl Acad Sci USA 2003, 100:2538-2543.

49. Richtsmeier Lab: Landmarks [http://www.getahead.psu.edu/ landmarks_new.html]

50. Fowles LF, Bennetts JS, Berkman JL, Williams E, Koopman P, Teasdale RD, Wicking C: Genomic screen for genes involved in mammalian craniofacial development. Genesis 2003, 35:73-87. 